(1)

CrossMark

\title{
The Electronic Asthma Management System (eAMS) improves primary care asthma management
}

\author{
Samir Gupta ${ }^{1,2}$, Courtney Price ${ }^{1}$, Gina Agarwal $^{3}$, David Chan ${ }^{3}$, Sanjeev Goel ${ }^{4}$, \\ Louis-Philippe Boulet ${ }^{5}$, Alan G. Kaplan ${ }^{6,7}$, Gerald Lebovic ${ }^{8,9}$, \\ Muhammad Mamdani ${ }^{10,11}$ and Sharon E. Straus ${ }^{1}$
}

@ERSpublications

The Electronic Asthma Management System is a systematically developed and evidence-based computerised decision support tool that engages both patients and healthcare providers to improve the quality of asthma care in real-world primary care settings http://ow.ly/w43Z30nxALB

Cite this article as: Gupta S, Price C, Agarwal G, et al. The Electronic Asthma Management System (eAMS) improves primary care asthma management. Eur Respir J 2019; 53: 1802241 [https://doi.org/ 10.1183/13993003.02241-2018].

ABSTRACT A high prevalence of suboptimal asthma control is attributable to known evidence-practice gaps. We developed a computerised clinical decision support system (the Electronic Asthma Management System (eAMS)) to address major care gaps and sought to measure its impact on care in adults with asthma.

This was a 2-year interrupted time-series study of usual care (year 1) versus eAMS (year 2) at three Canadian primary care sites. We included asthma patients aged $\geqslant 16$ years receiving an asthma medication within the last 12 months. The eAMS consisted of a touch tablet patient questionnaire completed in the waiting room, with real-time data processing producing electronic medical record-integrated clinician decision support.

Action plan delivery (primary outcome) improved from zero out of 412 (0\%) to 79 out of 443 (17.8\%) eligible patients (absolute increase 0.18 (95\% CI $0.14-0.22)$ ). Time-series analysis indicated a $30.5 \%$ increase in physician visits with action plan delivery with the intervention $(\mathrm{p}<0.0001)$. Assessment of asthma control level increased from 173 out of 3497 (4.9\%) to 849 out of 3062 (27.7\%) eligible visits (adjusted OR 8.62 (95\% CI 5.14-12.45)). Clinicians escalated controller therapy in 108 out of $3422(3.2 \%)$ baseline visits versus 126 out of $3240(3.9 \%)$ intervention visits $(p=0.12)$. At baseline, a short-acting $\beta$ agonist alone was added in 62 visits and a controller added in 54 visits; with the intervention, this occurred in 33 and 229 visits, respectively $(\mathrm{p}<0.001)$.

The eAMS improved asthma quality of care in real-world primary care settings. Strategies to further increase clinician uptake and a randomised controlled trial to assess impact on patient outcomes are now required.

This article has supplementary material available from erj.ersjournals.com

Received: Dec 052018 | Accepted after revision: Jan 232019

This study is registered at ClinicalTrials.gov with identifier number NCT01070095. Individual participant data that underlie the results reported in this article, after de-identification, as well as analytic code will be available immediately following article publication and ending 3 years following publication. Data requests will be considered from investigators whose proposed use of the data has been approved by our independent review committee for this purpose and for any proposed analysis. Proposals should be directed to the corresponding author; to gain access, data requestors will need to sign a data access agreement.

Copyright $\odot$ ERS 2019. This article is open access and distributed under the terms of the Creative Commons Attribution Non-Commercial Licence 4.0. 


\section{Introduction}

Asthma affects 339 million people globally and is increasing in prevalence [1]. Although well-controlled asthma is achievable in most patients, more than half of patients remain poorly controlled [2-4].

Three evidence-based care gaps are major contributors to poor asthma control [5, 6]. 1) Control monitoring: assessment of asthma control using guideline criteria is performed in as few as $1 \%$ of clinical encounters [7], resulting in under-recognition of suboptimal control [2, 8]. 2) Medication adjustment: only $39 \%$ of physicians report basing asthma therapy on guideline recommendations [8], leading to asthma under-treatment $[2,8] .3$ ) Asthma action plan (AAP) delivery: only $4 \%$ of primary care physicians report consistently providing a written AAP [9] and only $2 \%$ of asthma patients actually receive one [10].

Applying the Knowledge-to-Action Framework [11], we hypothesised that the underlying barriers to these key practices could be addressed by a point-of-care computerised clinical decision support system (CDSS). Given that the majority of patients with asthma are managed in primary care, we designed and integrated a CDSS (the Electronic Asthma Management System (eAMS)) into an electronic medical record (EMR) system. We sought to measure the impact of the eAMS on evidence-based care.

\section{Methods}

Study design, setting, population

This was a 2-year prospective interrupted time-series (ITS) design study of usual asthma care (baseline period) (year 1) followed by implementation of the eAMS (intervention period) (year 2). The Canadian study was approved by the St Michael's Hospital and Hamilton Integrated Research Ethics Boards, registered at ClinicalTrials.gov (identifier NCT01070095), and carried out across a convenience sample of two academic primary care sites in Hamilton, ON (population around 550000) (sites 1 and 2) and one nonacademic primary care site in Brampton, ON (population around 570000) (site 3). All prescribing clinicians were invited. We developed and validated an EMR search algorithm for asthma [12], and then applied this to the practices of consented clinicians. We included asthma patients aged $\geqslant 16$ years who understood English and had been on an asthma medication in the prior year. We excluded patients who had been on a chronic obstructive pulmonary disease medication in the prior year [12].

Further details are provided in the supplementary material.

\section{Intervention}

Overview

During the baseline period, all clinicians were e-mailed a link to an online educational module on how to complete an AAP and paper/electronic copies of a blank AAP. As reported previously, we developed the AAP for this trial through a systematic analysis of existing AAPs [13], patient and provider stakeholder wiki-based collaborative editing, and usability optimisation [14, 15]. During the intervention period, the eAMS was available in e-charts of all included patients (access was chart based and not clinician based). The eAMS consisted of: 1) a touch tablet patient questionnaire used in the clinic waiting room, and 2) a five-screen point-of-care CDSS that received and processed questionnaire data to produce decision support integrated into the EMR in real-time (figure 1). We developed the eAMS user interface through serial testing and user feedback, and implemented it through rounds presentations before and after launch, written pamphlets, and online resource materials.

\section{Patient questionnaire}

We developed the patient questionnaire and optimised content and usability through serial focus groups with asthma patients (described elsewhere [16, 17]), as previously recommended [18]. The questionnaire required 5-10 min to complete, and ascertained asthma control (using Canadian guideline-recommended

Affiliations: ${ }^{1}$ Keenan Research Centre, Li Ka Shing Knowledge Institute, St Michael's Hospital, Toronto, ON, Canada. ${ }^{2}$ Division of Respirology, Dept of Medicine, University of Toronto, Toronto, ON, Canada. ${ }^{3}$ Dept of Family Medicine, McMaster University, Hamilton, ON, Canada. ${ }^{4}$ Health Quality Innovation Collaborative, Brampton, ON, Canada. ${ }^{5}$ Institut Universitaire de Cardiologie et de Pneumologie de Québec, Universite Laval, Québec, QC, Canada. ${ }^{6}$ Family Physician Airways Group of Canada, Edmonton, AB, Canada. ${ }^{7}$ Respiratory Medicine Special Interest Focus Group, College of Family Physicians of Canada, Mississauga, ON, Canada. ${ }^{8}$ Applied Research Centre, Li Ka Shing Knowledge Institute, St Michael's Hospital, Toronto, ON, Canada. ${ }^{9}$ Institute for Health Policy Management and Evaluation, University of Toronto, Toronto, ON, Canada. ${ }^{10} \mathrm{Li} \mathrm{Ka}$ Shing Centre for Healthcare Analytics Research and Training (LKS-CHART), Li Ka Shing Knowledge Institute, St Michael's Hospital, Toronto, ON, Canada. ${ }^{11}$ Leslie Dan Faculty of Pharmacy, University of Toronto, Toronto, ON, Canada.

Correspondence: Samir Gupta, Keenan Research Centre, Li Ka Shing Knowledge Institute, Suite 6042, St Michael's Hospital, 30 Bond Street, Toronto, ON M5B 1W8, Canada. E-mail: guptas@smh.ca 


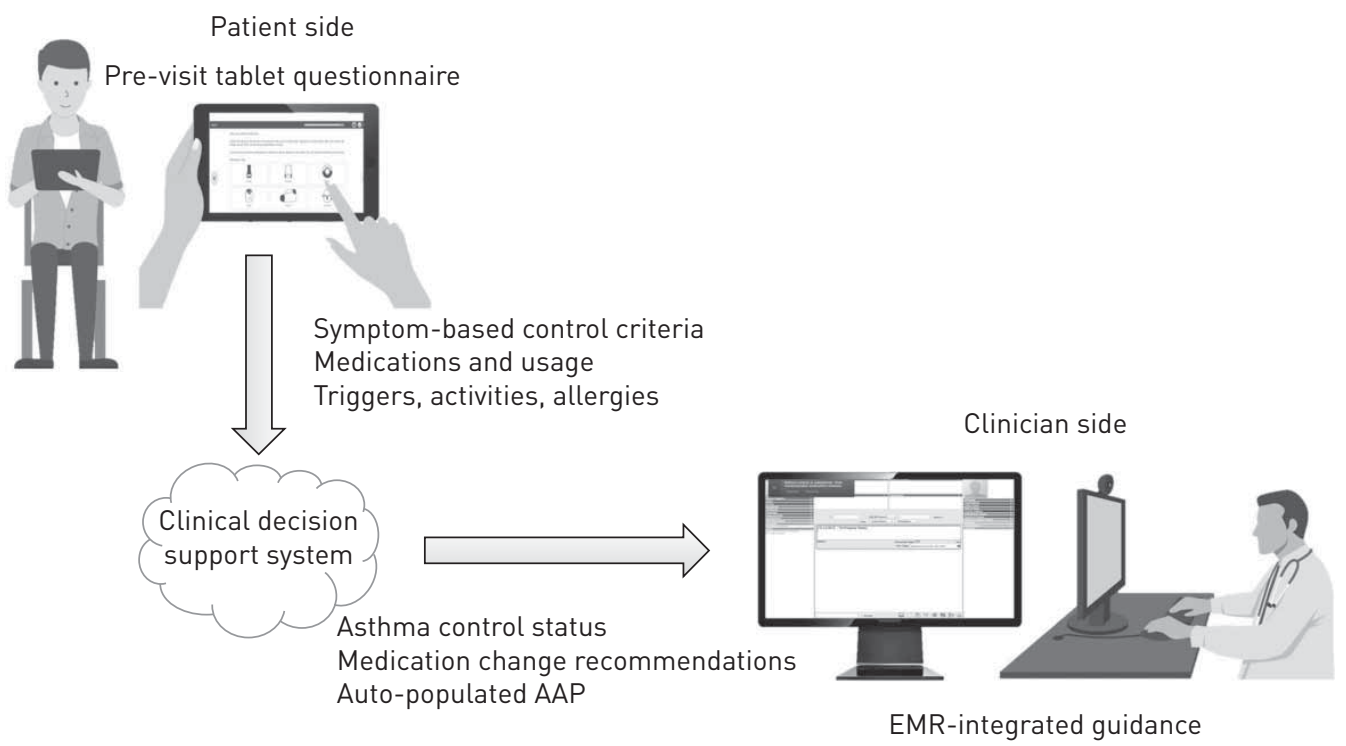

FIGURE 1 Electronic Asthma Management System (eAMS) schematic. EMR: electronic medical record; AAP: asthma action plan. The eAMS started with a touch tablet patient questionnaire used in the clinic waiting room, which collected asthma symptoms according to Canadian guideline-recommended criteria, medications and details required for AAP personalisation (patient-specific symptoms, activities, triggers and allergies). Next, these data were processed by an evidence-based computerised clinical decision support system in real-time, and guidance regarding asthma control status, corresponding medication change recommendations and an auto-populated, personalised AAP were then integrated into the clinician-facing EMR system.

symptom-based criteria) [19], medication use and details required for AAP personalisation (e.g. symptoms, activities (including sports), triggers and allergies). Patients were provided with the tablet questionnaire by a clinic staff member (e.g. receptionist) upon arrival for their appointment. An embedded questionnaire message encouraged patients to prompt their practitioner to provide an AAP.

\section{Clinical decision support system}

Upon opening the chart of any patient who had completed the questionnaire, clinicians saw a notification indicating the patient's current asthma control and prompting them to open the CDSS. The CDSS provided: the patient's control criteria and self-reported medication use (screen 1); evidence-based medication escalation/de-escalation recommendations (screen 2); AAP yellow zone (acute loss of control zone) medication recommendations (screen 3); a pre-populated AAP (screen 4); and a reminder to book follow-up and provide a pre-printed sticky note with an educational website URL (screen 5) (the website included inhaler technique videos and a self-directed web-based asthma educational programme) [20]. If users progressed through at least one of these screens, a documentation note was automatically written to the EMR chart. If clinicians approved the AAP, it was sent to the chart for printing.

The CDSS development process is described elsewhere [21-23]. This included development of medication escalation/de-escalation logic rules through a review of existing international asthma guidelines [21], establishment of evidence-based rules for AAP yellow zone medications through a systematic review [22], and application of evidence-based methods to optimise the language and format of provided guidance [23]. The AAP populated by the eAMS was the same as that used in the baseline phase (see earlier details).

Further intervention details are provided in the supplementary material.

\section{Outcomes}

Four trained reviewers performed an electronic chart audit of all outpatient visits and asthma-related telephone interactions (details in the supplementary material). The primary outcome was AAP delivery, measured by the proportion of patients/visits with written AAP delivery during the intervention versus baseline period (comparison of cumulative proportion of patients receiving an AAP; ITS analysis with autoregressive integrated moving average (ARIMA) model for visits with AAP delivery). Secondary outcomes included comparison of the cumulative proportion of patients/visits with asthma control determined according to symptom-based criteria (control determination required meeting one or more criteria for uncontrolled asthma or all criteria for controlled asthma; see supplementary table S1) and the 
proportion of patients/visits with escalation of controller therapy (including initiation of controller therapy) during the intervention versus baseline period. We attempted to identify predictors of these outcomes through a generalised linear mixed model (GLMM) including the following candidate parameters, defined a priori: clinic, appointment provider practitioner type, prior objective diagnosis of asthma, documented physician diagnosis of asthma, presenting complaint type, billing physician (most responsible physician/other), previous emergency department visit(s)/hospitalisation(s) for asthma and current asthma control. We compared the proportion of oral corticosteroid prescriptions and rescue to controller medication prescriptions between periods. During the intervention period, we measured the proportion of visits where patients had good control in which medications were de-escalated, the proportion of patients in whom discussions about medication adherence took place and system uptake.

\section{Analysis}

The intervention period included all visits that occurred while all components of the eAMS were active, in each respective clinic. We compared patient variables between baseline and intervention periods with Fisher's exact/Chi-squared tests and ANOVAs, as appropriate. We compared outcome proportions between periods with Chi-squared tests. AAP delivery measured the proportion of patients on an asthma controller medication for at least one visit who received an AAP (excluding those who had received/ reviewed an AAP in the last 6 months). In the ITS analysis, we compared outcomes rates in 26 consecutive 2 -week intervals prior to the intervention to those in 26 consecutive 2-week intervals following the intervention, using an ARIMA model. Model checking included autocorrelation and partial autocorrelation plots, and the Augmented Dicky-Fuller test and Ljung-Box test. In measuring asthma control assessment, we eliminated visits in which control had been assessed within 28 days [5]. In measuring therapy escalation, we eliminated visits in which escalation had been made within 3 months. To account for individual and clinical risk factors as well as the longitudinal component, we used a GLMM with a logit link to identify predictors of main outcomes among patients seen in both the baseline and intervention periods. A random effect was included to account for within-subject correlation (confidence intervals were bootstrapped). Analyses were performed using R version 3.4.0 (www.r-project.org). Statistical significance was defined at a two-sided 0.05 level. Sample size calculations and further analytical details are presented in the supplementary material.

\section{Results}

Chart review

Agreement between reviewers in chart abstraction was $82.8-97.3 \%$ for control criteria, $97.5 \%$ for assessment of medication changes and $100 \%$ for AAP delivery (the $\kappa$ statistic could not be computed due to perfect agreement for the primary outcome).

\section{Population}

We recruited 19 out of $37(51.4 \%)$ approached physicians and one out of three (33\%) approached nurse practitioners (NPs). One physician withdrew consent before intervention launch, leaving 18 out of 37 (48.4\%) physicians. The NP managed patients from an additional five physicians, enabling us to include patients from 23 out of $37(62.2 \%)$ physicians. These physicians had been in practice for $16.1 \pm 8.9$ years (mean \pm SD; range 2-33 years) and 15 out of 23 were female (65.2\%). They were the most responsible physician for 830 eligible patients seen in the baseline period (3565 eligible visits) and 890 seen in the intervention period (3444 eligible visits) (table 1). There were 1272 unique patients seen over the study period, with $382(30.0 \%)$ seen exclusively in the baseline period, 442 (34.8\%) seen exclusively in the intervention period and $448(35.2 \%)$ seen in both periods. Given that patients occasionally saw clinicians other than their most responsible physician for urgent issues, these patients received care from 237 unique providers: 156 residents, 60 staff physicians $(41$ (68.3\%) female, in practice for $16.6 \pm 11.8$ years (mean \pm SD; range 2-52)), $20 \mathrm{NPs}$ and three physician assistants. Each provider averaged 29.6 \pm 58.2 visits (mean \pm SD; range 1-439). In the baseline period, 140 out of 3565 (3.9\%) visits were related to asthma compared with 158 out of $3444(4.6 \%)$ visits in the intervention period $(\mathrm{p}=0.19)$ and 276 out of $830(33.3 \%)$ patients had at least one visit for a respiratory complaint compared with 281 out of 890 (31.6\%) in the intervention period $(\mathrm{p}=0.49)$.

\section{System uptake}

In the intervention period, 551 out of $890(61.2 \%)$ patients completed the questionnaire at least once. Clinicians accessed the CDSS in 174 of the 505 patients (34.4\%) in whom actions were required. A detailed mixed methods system uptake analysis will be reported separately. 


\begin{tabular}{|c|c|c|c|}
\hline Characteristic & Baseline period & Intervention period & p-value \\
\hline Subjects & 830 & 890 & \\
\hline Age years & $45.9 \pm 17.4$ & $47.3 \pm 17.2$ & 0.084 \\
\hline Female & $602(72.5)$ & $632(71.0)$ & 0.519 \\
\hline Smoking status & & & 0.001 \\
\hline Nonsmoker & $411(49.5)$ & $460(51.7)$ & \\
\hline Ex-smoker & $161(19.4)$ & $185(20.8)$ & \\
\hline Current smoker & $124(14.9)$ & $157(17.6)$ & \\
\hline Not documented & $134(16.1)$ & $88(9.9)$ & \\
\hline \multicolumn{4}{|l|}{ Comorbidities } \\
\hline Atopy & 331 (39.9) & 350 (39.3) & 0.285 \\
\hline COPD & $62(7.5)$ & $68(7.6)$ & 0.966 \\
\hline Other respiratory diagnosis & $16(1.9)$ & 13 (1.5) & 0.573 \\
\hline \multicolumn{4}{|l|}{ Prior objective testing } \\
\hline Spirometry & $529(63.7)$ & $579(65.1)$ & 0.600 \\
\hline Methacholine challenge & $74(8.9)$ & 73 (8.2) & 0.658 \\
\hline \multicolumn{4}{|l|}{ Prior asthma care (since 2003) } \\
\hline Seen by pulmonologist or allergist & $136(16.4)$ & $158(17.8)$ & 0.491 \\
\hline Seen in emergency room or hospitalised for asthma & $51(6.1)$ & $38(4.3)$ & 0.100 \\
\hline \multicolumn{4}{|l|}{ Baseline medications (first study visit) ${ }^{\#}$} \\
\hline Short-acting bronchodilator & $469(56.5)$ & $544(61.1)$ & 0.058 \\
\hline Inhaled corticosteroid alone $e^{\Uparrow}$ & $147(17.7)$ & $173(19.4)$ & 0.391 \\
\hline Inhaled corticosteroid with long-acting $\beta$-agonist & $125(15.1)$ & $158(17.8)$ & 0.150 \\
\hline Long-acting $\beta$-agonist alone & $6(0.7)$ & $4(0.4)$ & 0.669 \\
\hline Leukotriene receptor antagonist & $21(2.5)$ & $26(2.9)$ & 0.727 \\
\hline Long-acting muscarinic antagonist & $6(0.7)$ & $13(1.5)$ & 0.218 \\
\hline Prednisone $^{+}$ & $8(1.0)$ & $6(0.7)$ & 0.689 \\
\hline
\end{tabular}

Data are presented as $\mathrm{n}$, mean \pm SD or $\mathrm{n}(\%)$, unless otherwise stated. COPD: chronic obstructive pulmonary disease. ": formal assessment of asthma severity was not possible, as this requires knowledge of which medications are required to achieve good control and asthma control itself was not known for most patients la breakdown of baseline medications by control status is provided in the supplementary material); ๆ: without concurrent use of a long-acting $\beta$-agonist in a combination inhaler or as a separate inhaler; ${ }^{+}$: includes only those patients using prednisone chronically.

\section{Primary outcome}

The proportion of patients on a controller medication who received an AAP was zero out of $412(0 \%)$ in the baseline period and 79 out of $443(17.8 \%)$ in the intervention period (absolute increase 0.18 (95\% CI 0.14-0.22)). In the ITS analysis, there was an increase in the proportion of visits where patients received an AAP from zero at baseline to 0.305 at the time of intervention $(p<0.0001)$ (figure 2$)$. There was no significant decreasing trend as the intervention period progressed $(\mathrm{p}=0.17)$. Modelling was not possible due to the absence of AAP delivery in the baseline period.

\section{Secondary outcomes \\ Asthma control monitoring}

Practitioners determined asthma control according to symptom-based criteria in 173 out of 3497 (4.9\%) eligible visits in the baseline period and 849 out of $3062(27.7 \%)$ in the intervention period $(\mathrm{p}<0.001)$. Control was determined at least once in 118 out of $830(14.2 \%)$ and 523 out of 890 (58.8\%) patients, respectively $(\mathrm{p}<0.001)$. After adjusting for other variables, control was more likely to be ascertained during the intervention period (OR 8.62 (95\% CI 5.14-12.45)) (table 2).

\section{Controller therapy escalation}

Practitioners escalated controller therapy in 108 out of 3422 (3.2\%) eligible visits in the baseline period and 126 out of $3240(3.9 \%)$ in the intervention period $(\mathrm{p}=0.12)$. At least one therapeutic escalation occurred in 106 out of $830(12.8 \%)$ and 117 out of $890(13.1 \%)$ patients, respectively ( $\mathrm{p}=0.87)$. After adjusting for other variables, therapy was less likely to be escalated during the intervention period (OR 0.55 (95\% CI 0.28-0.99)) (table 3). In the baseline period, a short-acting $\beta$-agonist alone was added to therapy in 62 visits and a controller medication added in 54 visits (ratio 1.15) compared with 33 and 229 visits in the intervention period, respectively (ratio 0.14) $(\mathrm{p}<0.001)$. Oral corticosteroids were prescribed in 


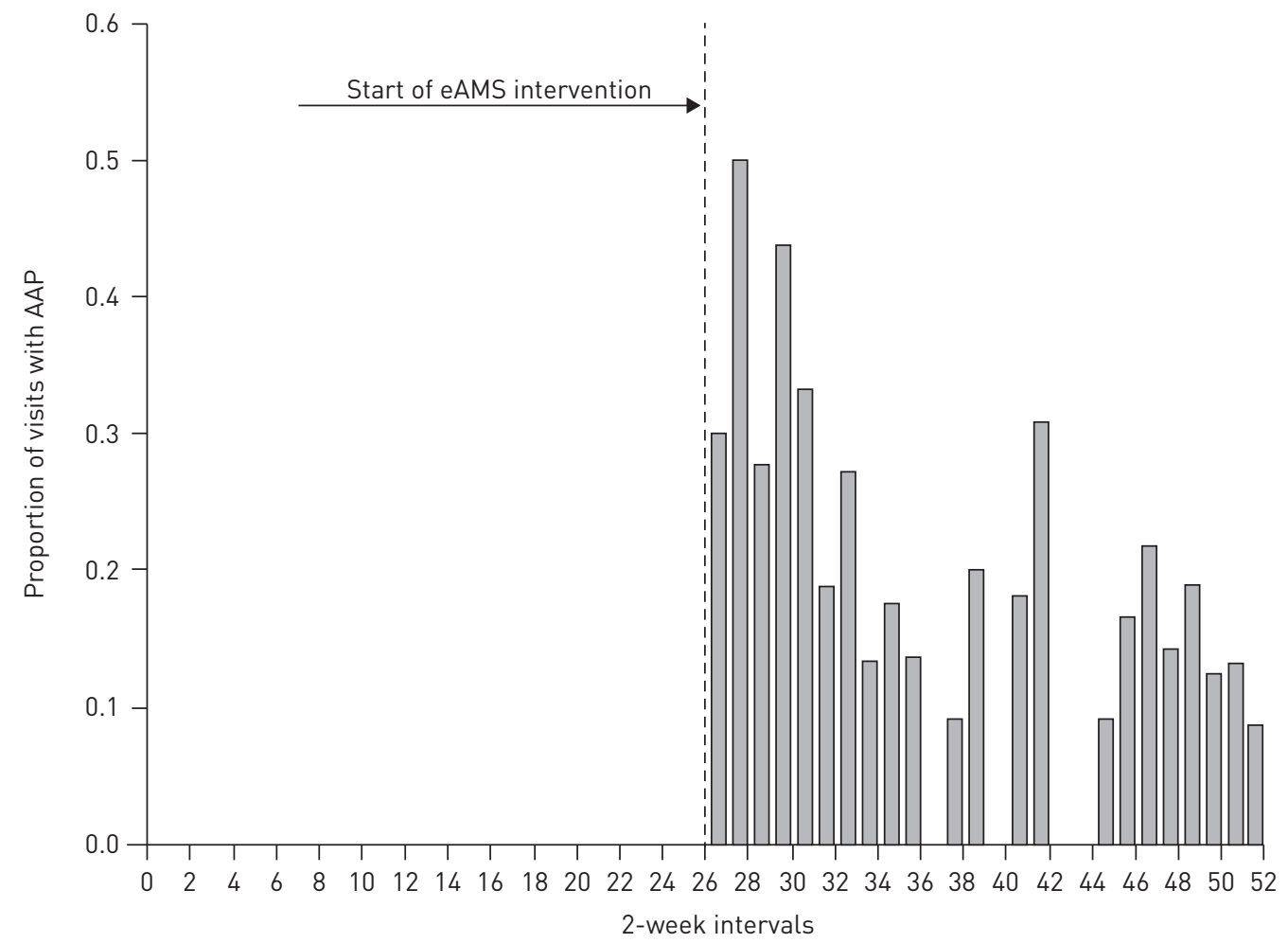

FIGURE 2 Time-series analysis. AAP: asthma action plan; eAMS: Electronic Asthma Management System. The proportion of asthma patient visits in which an AAP was delivered, at each 2-week interval (baseline period: intervals 0-26; intervention period: intervals 26-52). The arrow indicates the period in which the eAMS intervention was launched.

28 out of $3565(0.79 \%)$ visits and 24 out of $830(2.89 \%)$ patients in the baseline period, and in 16 out of $3444(0.46 \%)$ visits $(\mathrm{p}=0.089)$ and 15 out of $890(1.69 \%)$ patients $(\mathrm{p}=0.093)$ in the intervention period.

In the intervention period, clinicians de-escalated therapy in seven out of 459 (1.5\%) visits in which asthma control was adequate and documented medication adherence discussions in 219 out of 890 (24.6\%) patients.

\section{On-treatment analysis}

Counting only intervention period visits in which patients completed the questionnaire and the notification prompted clinicians to open the CDSS to take action, 81 out of 265 (30.6\%) patients received an AAP, control was assessed in 656 out of $770(85.2 \%)$ visits and medications were escalated in 69 out of $1001(6.9 \%)$ visits.

\section{Discussion}

We demonstrated that an evidence-based CDSS improves the quality of asthma care in real-world primary care settings while requiring minimal changes to local resources for successful implementation.

AAP delivery increased from zero at baseline to $17.8 \%$ of patients and $30.5 \%$ of visits with the intervention. Our nil baseline rate was comparable to the $2 \%$ rate described in a previous primary care chart audit [10]. Guidelines dating back to the 1990 British Asthma Guideline [24] and the 1996 Canadian Asthma Guideline [25] call for all patients to receive a written AAP. The majority of physicians consider AAPs important, but fail to provide them due to lack of time and confidence in generating AAP recommendations $[8,26]$. Although we previously published basic principles for populating the AAP yellow zone [22], experts suggested that an information technology approach would be essential to operationalising these in the real world [18]. Correspondingly, the eAMS targeted time and knowledge/ skill barriers by leveraging the CDSS to auto-populate the AAP. Studies have also shown that when AAPs are tailored and provided along with education, patients view them favourably and are more adherent [27]. The eAMS created a highly personalised AAP reflecting self-described symptoms, activities and triggers, and provided access to an asthma education programme. A primary care study identified the following enablers of AAP delivery: patients requesting an AAP, adding a blank AAP to the chart and receiving a 
TABLE 2 Predictors of asthma control assessment

\begin{tabular}{|c|c|c|c|}
\hline & $\begin{array}{l}\text { Control not } \\
\text { assessed }\end{array}$ & $\begin{array}{l}\text { Control } \\
\text { assessed }\end{array}$ & $\begin{array}{l}\text { GLMM model } \\
\text { OR }(95 \% \mathrm{CI})\end{array}$ \\
\hline Visits & 5537 & 1022 & $4143^{\#}$ \\
\hline \multicolumn{4}{|l|}{ Period ${ }^{\text {I }}$} \\
\hline Baseline & $3324(95.1)$ & $173(4.9)$ & \\
\hline Intervention & $2213(72.3)$ & $849(27.7)$ & $8.51(5.51-11.52)$ \\
\hline \multicolumn{4}{|l|}{ Clinic } \\
\hline 1 & 2290 (79.8) & $578(20.2)$ & \\
\hline 2 & 1052 (86.4) & $166(13.6)$ & $0.63(0.46-0.90)$ \\
\hline 3 & 2195 (88.8) & $278(11.2)$ & $0.28(0.21-0.41)$ \\
\hline \multicolumn{4}{|l|}{ Practitioner type } \\
\hline Physician & 2705 (84.9) & $481(15.1)$ & \\
\hline Nurse practitioner & $506(88.9)$ & $63(11.1)$ & $0.93(0.59-1.49)$ \\
\hline Resident & 1924 (82.0) & $423(18.0)$ & $1.38(0.99-1.86)$ \\
\hline Physician assistant & 402 (88.2) & $54(11.8)$ & $1.22(0.74-2.01)$ \\
\hline \multicolumn{4}{|l|}{ Objective asthma diagnosis } \\
\hline No & 3308 (85.2) & $576(14.8)$ & \\
\hline Yes & 2229 (83.3) & $446(16.7)$ & $0.78(0.61-0.99)$ \\
\hline \multicolumn{4}{|c|}{ Physician-documented asthma diagnosis } \\
\hline No & 2251 (88.8) & $283(11.2)$ & \\
\hline Yes & $3286(81.6)$ & $739(18.4)$ & $1.35(1.01-1.75)$ \\
\hline \multicolumn{4}{|l|}{ Presenting complaint } \\
\hline Asthma & $148(54.6)$ & $123(45.4)$ & \\
\hline Nonrespiratory & 4874 (87.7) & $684(12.3)$ & $0.11(0.08-0.18)$ \\
\hline Respiratory (nonasthma) & 515 (70.5) & $215(29.5)$ & $0.65(0.43-1.06)$ \\
\hline \multicolumn{4}{|c|}{$\begin{array}{l}\text { Previous emergency department visit(s) } \\
\text { /hospitalisation(s) }\end{array}$} \\
\hline No & $5278(84.8)$ & $948(15.2)$ & \\
\hline Yes & $259(77.8)$ & 74 (22.2) & $1.97(1.18-3.06)$ \\
\hline \multicolumn{4}{|c|}{ Seen by most responsible physician } \\
\hline No & 3676 (84.0) & $701(16.0)$ & \\
\hline Yes & 1861 (85.3) & $321(14.7)$ & $1.12(0.83-1.53)$ \\
\hline \multicolumn{4}{|c|}{ 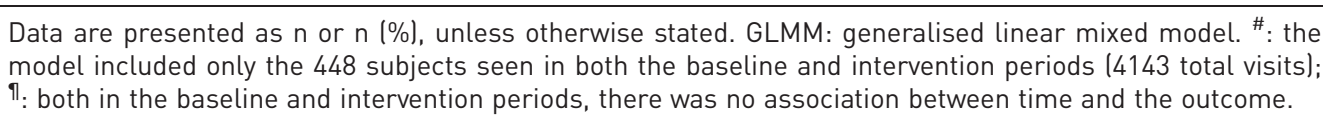 } \\
\hline
\end{tabular}

completed copy of the AAP [9]. Indeed, the eAMS questionnaire asked patients to prompt their practitioners to provide them with the AAP and the CDSS auto-completed a blank AAP for clinicians.

Asthma control monitoring also improved with the intervention. Control was assessed in $4.9 \%$ of baseline visits, comparable to a US primary care chart review in which all control criteria were assessed in $1 \%$ of patients [7]. In a UK review, only $27 \%$ patients who died of asthma had had control assessed at their most recent primary care visit [28]. Improving control monitoring could improve health-related quality of life, avoid lost productivity and reduce healthcare costs through better disease management and adherence [29], although barriers are multi-fold. Physicians often pose an open-ended control question (e.g. "How is your asthma") [8]. However, most patients are unaware of their poor control. In a European survey of 8000 patients with asthma, $20.1 \%$ had controlled asthma, yet $90.5 \%$ self-identified as well controlled [4]. Correspondingly, a failure to probe each control parameter leads to under-reporting of poor control [8]. Physicians may also overestimate control. In a practice-based assessment, physicians labelled $31 \%$ of their inadequately controlled patients as "well controlled" [2]. Failure to assess control objectively may be due to lack of familiarity with criteria. Canadian primary care physicians identified an average of 2.2 out of eight control criteria [30] and $26 \%$ of US primary care physicians were confident that they could assess asthma control [7]. Additional barriers include lack of time and simply forgetting to assess control [30]. The eAMS attempted to address these barriers by posing control questions in the patient questionnaire and "pushing" results to clinicians.

We did not identify a higher proportion of controller medication escalations with the intervention. The eAMS attempted to address known barriers to appropriate adjustment, including failure to recognise poor control and lack of knowledge regarding asthma pharmacotherapeutics [31]. However, patient factors including inhaled corticosteroid aversion and affordability may also play a role [8]. We noted that 


\begin{tabular}{|c|c|c|c|}
\hline & $\begin{array}{l}\text { Controller } \\
\text { escalation not made }\end{array}$ & $\begin{array}{c}\text { Controller } \\
\text { escalation made }\end{array}$ & $\begin{array}{l}\text { GLMM model } \\
\text { OR }(95 \% \mathrm{CI})\end{array}$ \\
\hline Visits & 6428 & 234 & $4204^{\#}$ \\
\hline \multicolumn{4}{|l|}{ Period ${ }^{\pi}$} \\
\hline Baseline & $3314(96.8)$ & 108 (3.2) & \\
\hline Intervention & $3114(96.1)$ & $126(3.9)$ & $0.55(0.28-0.99)$ \\
\hline \multicolumn{4}{|l|}{ Clinic } \\
\hline 1 & 2947 (97.7) & $67(2.3)$ & \\
\hline 2 & $1244(97.6)$ & $31(3.4)$ & $0.82(0.42-1.44)$ \\
\hline 3 & 2237 (94.3) & $136(5.7)$ & $2.19(1.51-3.53)$ \\
\hline \multicolumn{4}{|l|}{ Practitioner type } \\
\hline Physician & $3087(96.0)$ & $130(4.0)$ & \\
\hline Nurse practitioner & 547 (96.5) & $20(3.5)$ & $0.82(0.38-1.74)$ \\
\hline Resident & $2368(97.3)$ & $66(2.7)$ & $0.79(0.46-1.41)$ \\
\hline Physician assistant & 425 (95.9) & $18(4.1)$ & $0.55(0.19-1.16)$ \\
\hline \multicolumn{4}{|l|}{ Objective asthma diagnosis } \\
\hline No & 3736 (95.9) & $158(4.1)$ & \\
\hline Yes & 2692 (97.3) & $76(2.7)$ & $0.46(0.31-0.68)$ \\
\hline \multicolumn{4}{|c|}{ Physician-documented asthma diagnosis } \\
\hline No & 2507 (97.7) & $60(2.3)$ & \\
\hline Yes & 3921 (95.8) & $174(4.2)$ & $1.35(0.90-2.05)$ \\
\hline \multicolumn{4}{|l|}{ Presenting complaint } \\
\hline Asthma & $201(76.4)$ & $62(23.6)$ & \\
\hline Nonrespiratory & $5610(98.5)$ & $85(1.5)$ & $0.06(0.04-0.10)$ \\
\hline Respiratory (nonasthma) & $617(87.6)$ & $87(12.4)$ & $0.51(0.33-0.85)$ \\
\hline \multicolumn{4}{|c|}{$\begin{array}{l}\text { Previous emergency department visit(s) } \\
\text { /hospitalisation(s) }\end{array}$} \\
\hline No & $6096(96.5)$ & $221(3.5)$ & \\
\hline Yes & $332(96.2)$ & $13(3.8)$ & $1.18(0.36-2.25)$ \\
\hline \multicolumn{4}{|c|}{ Seen by most responsible physician } \\
\hline No & $4308(96.6)$ & $151(3.4)$ & \\
\hline Yes & $2120(96.2)$ & $83(3.8)$ & $0.89(0.58-1.45)$ \\
\hline \multicolumn{4}{|c|}{ Current level of asthma control } \\
\hline Not documented & 4587 (97.5) & $117(2.5)$ & \\
\hline Poor control & $1302(92.4)$ & $107(7.6)$ & $2.23(1.52-3.30)$ \\
\hline Good control & $539(98.2)$ & $10(1.8)$ & $0.52(0.09-1.15)$ \\
\hline
\end{tabular}

Data are presented as $\mathrm{n}$ or $\mathrm{n}(\%)$, unless otherwise stated. GLMM: generalised linear mixed model. * : the model included only the 448 subjects seen in both the baseline and intervention periods ( 4204 total visits);

ๆ: both in the baseline and intervention periods, there was no association between time and the outcome.

presenting clinicians with patient-reported medication use (CDSS screen 1) led to discussions about adherence in $24.6 \%$ of patients. Given reported controller adherence rates of only 30-40\% [32], it is conceivable that adherence counselling discussions obviated the need for medication escalation in many patients. There was an improvement in the ratio of rescue to controller medication prescriptions in the intervention period (a metric associated with reduced healthcare utilisation and systemic steroid requirements) [33]. This suggests that although the quantity of prescriptions did not improve, their quality may have. We also note that therapeutic de-escalations occurred in only $1.5 \%$ of visits in which asthma control was adequate, despite eAMS recommendations for de-escalation. Determinants of de-implementation may be different than those of implementation and require further study.

Other asthma care CDSSs have been reported. Between two 2014 systematic reviews of trials of airways disease-related e-health interventions, we identified three included studies describing EMR-integrated clinician-facing CDSSs that provided complex patient-tailored decision support (beyond simple reminders) for nonemergent adult asthma management [34, 35]. TIERnEY et al.'s [36] system used EMR data to generate suggestions regarding immunisation, prescriptions and smoking cessation (no AAP), and showed no effects on guideline-based care. KUILBOER et al.'s [37] system used EMR data to generate critiques of physician treatments (no AAP), demonstrating increased lung function testing and reduced cromogylate prescriptions. Finally, PLAZA et al.'s [38] system used chart and clinician-entered data to determine asthma control and provide corresponding medication recommendations (no AAP). They demonstrated improved pharmacotherapy and symptoms, but results were confounded by a concurrent nurse education 
programme [38]. More recently, TAMBLYN et al. [39] reported a system comparable to ours that assessed control, provided medication recommendations and auto-populated an AAP. The system estimated control on the basis of rescue medication refills and healthcare utilisation from administrative databases, requiring clinician entry for other control criteria. In a randomised controlled trial, the primary outcome (out-of-control asthma events) did not differ between groups, although as in our study the ratio of short-acting $\beta$-agonist to controller medication prescriptions improved [39]. KuHN et al. [40] described an electronic AAP that required physician activation and data entry. In an observational study, an "on-treatment" analysis suggested lower oral corticosteroid requirements and exacerbations among children (but not adults). Although these two studies provided an auto-populated AAP, neither used a validated or personalised AAP designed with human factors optimisation (as our tool does). Furthermore, none of the aforementioned systems engaged patients to reduce data collection and entry burdens on clinicians (addressed through the pre-visit questionnaire in our tool), therefore failing to address clinician time constraints at the point of care [40]. Qualitative studies confirm that even when CDSSs improve care, time constraints are a critical barrier to their usage [41].

Clinicians accessed the eAMS in $34.4 \%$ of patients in whom actions were required. This uptake rate compares favourably with that of previous asthma CDSSs [35]. Among evidence-based predictors of successful CDSSs presented in the recent GUIDES checklist [42], the eAMS includes automatic prompting (a "push" strategy), "just in time" information delivery (during the clinical visit), clinical workflow integration (integration with existing EMR), patient-targeted information, patient-mediated clinician prompts and individualised recommendations. Strategies to drive improvement may include formal usability and workflow analysis and optimisation [42], features to address multiple morbidities, prompts/ advice that can be tailored to clinician preferences, detailed explanations/references for recommendations, and a requirement that practitioners supply a reason for over-riding advice [42].

Our study has several limitations. Measurements of control assessment may have been underestimated due to poor chart documentation. However, we believe that clinicians would be very likely to document poor control if ascertained, given its clinical relevance and influence on treatment decisions. An ITS design is vulnerable to temporal factors affecting behaviour. To the best of our knowledge, no such changes (e.g. changes in payment models, new educational programmes or allied health support) occurred during the study. We chose behavioural outcomes based on guideline recommendations and known correlations to patient outcomes, but did not directly measure patient outcomes.

In summary, we describe improvements in the quality of asthma care after implementation of a complex e-health intervention in a real-world setting. System improvements may be considered to further drive uptake and a future randomised controlled trial should assess impact on patient-level outcomes.

Acknowledgements: We would like to thank David Newton, Nicolas Leblanc and Susan Hall (Keenan Research Centre, Li Ka Shing Knowledge Institute, St Michael's Hospital, Toronto, ON, Canada) for their invaluable contributions to this work. We also acknowledge the support provided by the Asthma Society of Canada, the Canadian Thoracic Society, the Ontario Lung Association, and the Ontario Ministry of Health and Long-Term Care.

Author contributions: S. Gupta conceived of the study, oversaw all aspects of the study and prepared the manuscript; C. Price contributed to the data cleaning, analysis and interpretation; G. Agarwal, D. Chan and S. Goel contributed to study design, recruitment and manuscript revisions; S.E. Straus, A.G. Kaplan and L-P. Boulet contributed to study design and manuscript revisions; M. Mamdani and G. Lebovic contributed to study design, statistical analysis and interpretation, and manuscript revisions.

Conflict of interest: S. Gupta has nothing to disclose. C. Price has nothing to disclose. G. Agarwal has nothing to disclose. D. Chan has nothing to disclose. S. Goel has nothing to disclose. L-P. Boulet reports grants from AstraZeneca, Boston Scientific, GlaxoSmithKline, Hoffman La Roche, Novartis, Ono Pharma, Sanofi and Takeda, support for research projects from AstraZeneca, Boehringer Ingelheim, GlaxoSmithKline, Merck and Takeda, consultancy and advisory board work for AstraZeneca, Novartis and Methapharm, grants for production of educational materials from AstraZeneca, Boehringer Ingelheim, GlaxoSmithKline, Merck Frosst and Novartis, conference fees from AstraZeneca, GlaxoSmithKline, Merck and Novartis, support for participation in conferences and meetings from Novartis and Takeda; is past president and member of the Canadian Thoracic Society Respiratory Guidelines Committee; chair of the board of directors of the Global Initiative for Asthma (GINA); chair of GINA guidelines dissemination and implementation committee; Laval University chair on knowledge transfer, prevention and education in respiratory and cardiovascular health; member of scientific committees for the American College of Chest Physicians, American Thoracic Society, European Respiratory Society and the World Allergy Organization; First Vice-President of the global asthma organisation "Interasma". A.G. Kaplan has nothing to disclose. G. Lebovic has nothing to disclose. M. Mamdani reports advisory board work for NovoNordisk, Allergan and Neurocrine, and has lectured at an educational event for Amgen, outside the submitted work. S.E. Straus has nothing to disclose.

Support statement: This work was supported by the Canadian Institutes of Health Research (236225 and 322013). S. Gupta is supported by the Michael Locke Chair in Knowledge Translation and Rare Lung Disease Research. S.E. Straus is supported by a Tier 1 Canada Research Chair in Knowledge Translation and the Mary Trimmer Chair in Geriatric Medicine. Funding sources had no role in study design; in the collection, analysis and interpretation of data; in 
the writing of the report; or in the decision to submit the paper for publication. Funding information for this article has been deposited with the Crossref Funder Registry.

\section{References}

1 GBD 2016 Disease and Injury Incidence and Prevalence Collaborators. Global, regional, and national incidence, prevalence, and years lived with disability for 328 diseases and injuries for 195 countries, 1990-2016: a systematic analysis for the Global Burden of Disease Study 2016. Lancet 2017; 390: 1211-1259.

2 Chapman KR, Boulet LP, Rea RM, et al. Suboptimal asthma control: prevalence, detection and consequences in general practice. Eur Respir J 2008; 31: 320-325.

3 Sullivan PW, Ghushchyan V, Navaratnam P, et al. National prevalence of poor asthma control and associated outcomes among school-aged children in the United States. J Allergy Clin Immunol Pract 2018; 6: 536-544.

4 Price D, Fletcher M, van der Molen T. Asthma control and management in 8,000 European patients: the REcognise Asthma and LInk to Symptoms and Experience (REALISE) survey. NPJ Prim Care Respir Med 2014; 24: 14009.

5 To T, Guttmann A, Lougheed MD, et al. Evidence-based performance indicators of primary care for asthma: modified RAND Appropriateness Method. Int J Qual Health Care 2010; 22: 476-485.

6 National Institute for Health and Care Excellence. Asthma: Quality Statements. 2018. www.nice.org.uk/guidance/ qs25/chapter/Quality-statements Date last accessed: November 25, 2018.

7 Cicutto L, Dingae MB, Langmack EL. Improving asthma care in rural primary care practices: a performance improvement project. J Contin Educ Health Prof 2014; 34: 205-214.

8 FitzGerald JM, Boulet LP, McIvor RA, et al. Asthma control in Canada remains suboptimal: the Reality of Asthma Control (TRAC) study. Can Respir J 2006; 13: 253-259.

9 Djandji F, Lamontagne AJ, Blais L, et al. Enablers and determinants of the provision of written action plans to patients with asthma: a stratified survey of Canadian physicians. NPJ Prim Care Respir Med 2017; 27: 21.

10 Tsuyuki RT, Sin DD, Sharpe HM, et al. Management of asthma among community-based primary care physicians. J Asthma 2005; 42: 163-167.

11 Graham ID, Logan J, Harrison MB, et al. Lost in knowledge translation: time for a map? J Contin Educ Health Prof 2006; 26: 13-24.

$12 \mathrm{Xi}$ N, Wallace R, Agarwal G, et al. Identifying patients with asthma in primary care electronic medical record systems: a chart analysis-based electronic algorithm validation study. Can Fam Physician 2015; 61: e474-e483.

13 Gupta S, Wan FT, Ducharme FM, et al. Asthma action plans are highly variable and do not conform to best visual design practices. Ann Allergy Asthma Immunol 2012; 108: 260-265.

14 Gupta S, Wan F, Newton D, et al. WikiBuild: a new online collaboration process for multistakeholder tool development and consensus building. J Med Internet Res 2011; 13: e108.

15 Gupta S, Wan FT, Hall SE, et al. An asthma action plan created by physician, educator and patient online collaboration with usability and visual design optimization. Respiration 2012; 84: 406-415.

16 Gupta S, Lam Shin Cheung V, Kastner M, et al. Patient preferences for a touch screen tablet-based asthma questionnaire. J Asthma 2018; in press [https://doi.org/10.1080/02770903.2018.1490750].

17 Lam Shin Cheung V, Kastner M, Sale JE, et al. Development process and patient usability preferences for a touch screen tablet-based questionnaire. Health Informatics J 2019; in press [https://oi.org/10.1177/1460458218824749].

18 Bousquet J, Chavannes $\mathrm{NH}$, Guldemond $\mathrm{N}$, et al. Realising the potential of mHealth to improve asthma and allergy care: how to shape the future. Eur Respir J 2017; 49: 1700447.

19 Lougheed MD, Lemiere C, Dell SD, et al. Canadian Thoracic Society Asthma Management Continuum - 2010 Consensus Summary for children six years of age and over, and adults. Can Respir J 2010; 17: 15-24.

20 Gibson PG, Coughlan J, Wilson AJ, et al. Self-management education and regular practitioner review for adults with asthma. Cochrane Database Syst Rev 2003; 1: CD001117.

21 Gupta S, Paolucci E, Kaplan A, et al. Contemporaneous international asthma guidelines present differing recommendations: an analysis. Can Respir J 2016; 2016: 3085065

22 Kouri A, Boulet LP, Kaplan A, et al. An evidence-based, point-of-care tool to guide completion of asthma action plans in practice. Eur Respir J 2017; 49: 1602238.

23 Gupta S, Rai N, Bhattacharrya $\mathrm{O}$, et al. Optimizing the language and format of guidelines to improve guideline uptake. CMAJ 2016; 188: E362-E368.

24 British Thoracic Society, Research Unit of the Royal College of Physicians of London, King's Fund Centre, et al. Guidelines for management of asthma in adults: I - chronic persistent asthma. Br Med J 1990; 301: 651-653.

25 Ernst P, Fitzgerald JM, Spier S. Canadian asthma consensus conference summary of recommendations. Can Respir J 1996; 3: 89-101.

26 Labelle $\mathrm{M}$, Beaulieu M, Renzi $\mathrm{P}$, et al. Integrating clinical practice guidelines into daily practice: impact of an interactive workshop on drafting of a written action plan for asthma patients. J Contin Educ Health Prof 2004; 24: 39-49.

27 Ring N, Malcolm C, Wyke S, et al. Promoting the use of personal asthma action plans: a systematic review. Prim Care Respir J 2007; 16: 271-283.

28 Royal College of Physicians. Why Asthma Still Kills: The National Review of Asthma Deaths (NRAD) Confidential Enquiry Report. London, RCP, 2014

29 Sadatsafavi $\mathrm{M}$, Chen $\mathrm{W}$, Tavakoli $\mathrm{H}$, et al. Saving in medical costs by achieving guideline-based asthma symptom control: a population-based study. Allergy 2016; 71: 371-377.

30 Renzi PM, Ghezzo H, Goulet S, et al. Paper stamp checklist tool enhances asthma guidelines knowledge and implementation by primary care physicians. Can Respir J 2006; 13: 193-197.

31 Goeman DP, Hogan CD, Aroni RA, et al. Barriers to delivering asthma care: a qualitative study of general practitioners. Med J Aust 2005; 183: 457-460.

32 Sumino K, Cabana MD. Medication adherence in asthma patients. Curr Opin Pulm Med 2013; 19: 49-53.

33 Laforest L, Licaj I, Devouassoux G, et al. Prescribed therapy for asthma: therapeutic ratios and outcomes. BMC Fam Pract 2015; 16: 49.

34 Fathima M, Peiris D, Naik-Panvelkar P, et al. Effectiveness of computerized clinical decision support systems for asthma and chronic obstructive pulmonary disease in primary care: a systematic review. BMC Pulm Med 2014; 14: 189. 
35 Matui P, Wyatt JC, Pinnock H, et al. Computer decision support systems for asthma: a systematic review. NPJ Prim Care Respir Med 2014; 24: 14005.

36 Tierney WM, Overhage JM, Murray MD, et al. Can computer-generated evidence-based care suggestions enhance evidence-based management of asthma and chronic obstructive pulmonary disease? A randomized, controlled trial. Health Serv Res 2005; 40: 477-497.

37 Kuilboer MM, van Wijk MA, Mosseveld M, et al. Feasibility of AsthmaCritic, a decision-support system for asthma and COPD which generates patient-specific feedback on routinely recorded data in general practice. Fam Pract 2002; 19: 442-447.

38 Plaza V, Cobos A, Ignacio-Garcia JM, et al. Coste-efectividad de una intervencion basada en las recomendaciones de la Global INitiative for Asthma (GINA), mediante un sistema informatizado de apoyo a la decision clinica: un ensayo con aleatorizacion de medicos. [Cost-effectiveness of an intervention based on the Global INitiative for Asthma (GINA) recommendations using a computerized clinical decision support system: a physicians randomized trial.] Med Clin 2005; 124: 201-206.

39 Tamblyn R, Ernst P, Winslade N, et al. Evaluating the impact of an integrated computer-based decision support with person-centered analytics for the management of asthma in primary care: a randomized controlled trial. J Am Med Inform Assoc 2015; 22: 773-783.

40 Kuhn L, Reeves K, Taylor Y, et al. Planning for action: the impact of an asthma action plan decision support tool integrated into an electronic health record (EHR) at a large health care system. J Am Board Fam Med 2015; 28: 382-393.

41 Gadd CS, Baskaran P, Lobach DF. Identification of design features to enhance utilization and acceptance of systems for Internet-based decision support at the point of care. Proc AMIA Symp 1998; 1998: 91-95.

42 Van de Velde S, Kunnamo I, Roshanov P, et al. The GUIDES checklist: development of a tool to improve the successful use of guideline-based computerised clinical decision support. Implement Sci 2018; 13: 86. 